\title{
Implementing the plasma-lasing potential for tabletop nano-imaging
}

\author{
Mabel Ruiz-Lopez $\cdot$ Davide Bleiner
}

Received: 11 January 2013/Accepted: 6 August 2013/Published online: 27 August 2013

(C) Springer-Verlag Berlin Heidelberg 2013

\begin{abstract}
Implementing the plasma-lasing potential for tabletop nano-imaging on across a hot plasma medium drives short-wavelength lasing, promising for "turnkey" nano-imaging setups. A systematic study of the illumination characteristics, combined with design-adapted objectives, is presented. It is shown how the ultimate nano-scale feature is dictated by either the diffraction-limited or the wavefront-limited resolution, which imposed a combined study of both the source and the optics. For nano-imaging, the spatial homogeneity of the illumination (spot noise) was shown as critical. Plasma-lasing from a triple grazingincidence pumping scheme compensated for the missing spot homogeneity in classical schemes. We demonstrate that a collimating mirror pre-conditions both the pointing stability and the divergence below half a mrad.
\end{abstract}

\section{Introduction}

The use of short-wavelength illumination is crucial for the progress of microscopy and for its enabling character, i.e., beating the micrometer resolution limit of present day tabletop systems, and accessing imaging at the nano-scale in the own laboratory. It is well known that shorter wavelengths push down the diffraction limit, since the ultimate resolved structure is given by $0.61 \lambda / N A$, with $\lambda$ the illumination wavelength and $N A$ the numerical aperture of the imaging system. In commercial microscopes working in the visible, the ultimate resolution is $>>500 \mathrm{~nm}$. For contrast, using $\lambda=10-100 \mathrm{~nm}$ available from plasma

M. Ruiz-Lopez · D. Bleiner $(\bowtie)$

Institute of Applied Physics, University of Bern,

Sidlerstrasse 5, 3012 Bern, Switzerland

e-mail: bleiner@iap.unibe.ch sources, a few research groups have demonstrated proofof-principle extreme ultraviolet (EUV) microscopy systems, with resolutions down to $50-80 \mathrm{~nm}[1,2]$. Such nano-imaging systems have been proposed for enabling actinic nano-inspection of EUV lithography masks, as shown at synchrotrons [3], or for cutting-edge material science applications [4], in the own laboratory. Optimization and high-duty research are, however, not possible at synchrotron sources, due to the limited and discontinuous accessibility. Tabletop systems are therefore desirable.

The basic elements of a microscope are the following ones: (1) photon source, (2) sample holder, (3) objective and (4) detector. Our study was restricted to the light source and the imaging optics, since CCDs in the X-ray and EUV ranges are well-established compact, and commercially available, components. On the other hand, shortwavelength compact photon sources as well as the related multilayer optics are still subject of fundamental research, which we believe should not be carried out separately, in order to provide integrated specifications for turnkey systems.

Most of the reported nano-inspection systems have been demonstrated in conjunction with large-footprint sources, such as the synchrotron [3]. The miniaturization of the EUV sources for own laboratory operation and daily access is a technology-enabling achievement, if happens without deterioration of the state-of-art performance. The EUV laser (also known as "X-ray laser" or XRL), generated using a laser-plasma gain-medium, is a well-debated platform for enabling nano-scale microscopy in the own laboratory. Amplified spontaneous emission (ASE) along the plasma column leads to as high as $10^{11}$ coherent photons, i.e., $1-10 \mu \mathrm{J}$ at wavelengths as low as $8-23 \mathrm{~nm}$ (depending on the target material), with just 1-10 Joules of pump energy on the target. Such pump energies are nowadays 
Table 1 Typical specifications for short-wavelength plasma-laser [6, 39], capillary discharge [40, 41] and X-ray free-electron laser [11, 44]

\begin{tabular}{|c|c|c|c|c|c|}
\hline Source & \multicolumn{2}{|l|}{ Plasma-laser } & \multicolumn{2}{|c|}{ Capillary discharge [40-42] } & Free electron laser [11] \\
\hline Divergence & \multicolumn{2}{|l|}{$5 \mathrm{mrad}$} & \multicolumn{2}{|c|}{$7-14 \mathrm{mrad}$} & $1-1.4 \mathrm{nrad}$ \\
\hline Pointing stability & \multicolumn{2}{|c|}{$2.2-3.6 \mathrm{mrad}[39]$} & \multicolumn{2}{|c|}{$25 \mathrm{mrad}$} & $0.25 \mathrm{urad}$ \\
\hline Spatial coherence & \multicolumn{2}{|l|}{$37 \%[10]$} & \multicolumn{2}{|c|}{$\sim 100 \%$} & $100 \%$ \\
\hline Relative bandwidth & \multicolumn{2}{|l|}{$10^{-4}[6]$} & \multicolumn{2}{|c|}{$10^{-4}$} & $10^{-3}$ \\
\hline Peak brilliance & \multicolumn{2}{|c|}{$10^{27} \mathrm{ph} / \mathrm{s} \cdot \mathrm{mm}^{2} \cdot \mathrm{mrad}^{2} 0.1 \% \mathrm{BW}[6]$} & \multicolumn{2}{|c|}{$2.10^{26} \mathrm{ph} / \mathrm{s} \cdot \mathrm{mm}^{2} \cdot \mathrm{mrad}^{2} 0.1 \% \mathrm{BW}$} & $10^{30} \mathrm{ph} / \mathrm{s} \cdot \mathrm{mm}^{2} \cdot \mathrm{mrad}^{2} 0.1 \% \mathrm{BW}$ \\
\hline Wavelength & \multicolumn{2}{|c|}{ Soft X-Ray / EUV [16] } & \multicolumn{2}{|c|}{ EUV } & Hard X-Ray \\
\hline \multicolumn{2}{|l|}{ Objective } & \multicolumn{2}{|c|}{ Schwarzschild (This work) } & Schwarzschild (Aachen) [25] & Fresnel zone plate (Colorado) \\
\hline \multicolumn{2}{|c|}{ Spherical aberration (Seidel Coeff.) } & $<0.001$ & & $<0.001$ & $<0.001$ \\
\hline \multicolumn{2}{|l|}{ NA } & 0.15 & & 0.22 & $0.066[2]$ \\
\hline \multicolumn{2}{|l|}{ Obscuration } & $17 \%$ & & $15-18 \%$ & None \\
\hline \multicolumn{2}{|l|}{ Magnification } & $30 \times$ & & $21.34 \times$ & $660 \times[2]$ \\
\hline \multicolumn{2}{|l|}{ Efficiency } & $17.2 \%$ & & $20 \%$ & $10 \%[44]$ \\
\hline \multicolumn{2}{|l|}{ Resolution } & See Table 3 & & $<100 \mathrm{~nm}$ & 54 nm [44] \\
\hline
\end{tabular}

In the bottom part, three different microscope objectives are compared: two different Schwarzschild designs (our group and Aachen)[26] and Fresnel zone plates at Colorado State University $[2,43]$

easy to achieve on tabletop setups, for instance by means of chirped-pulse amplification [5, 6]. The plasma-laser is more performing when pump pre-pulses on the solid target are used for generating a pre-plasma, then irradiated with a main pump pulse inducing ASE conditions across a hot/ dense plasma column. The decoupling of the ion-ensembleformation and their population inversion permits more flexibility in the optimization. The optimization of prepulse delivery is indeed subject of ongoing research, in order to enhance the conversion efficiency as well as the laser output characteristics [7]. Transient collisional excitation (TCE) [8] is accomplished with a $0.5-0.8 \mathrm{~ns}$ prepulse, orthogonal to the target, followed within its temporal duration by a short $10-50$ ps main pulse. Grazing-incidence pumping (GRIP) [9] showed further improvements delivering the short main pulse at $10^{\circ}-30^{\circ}$ grazing incidence. Our laboratory introduced a dual pre-pulse followed by the main pulse all at GRIP (TGRIP) [10]. This specific geometry of the triple GRIP permits a more accurate alignment of the pre-pulses with the main pulse. The use of two pre-pulses of good pedestal contrast permitted a stricter control of the atomization, ionization and population inversion stages. Whether the TGRIP scheme proves to be more suitable than classical TCE for imaging application in terms of profile and reproducibility is still not addressed in the literature and is subject of this work.

Further, the reproducibility of the illumination is a critical aspect in any microscopy source. In particular, this applies to a nano-science system, where any minimal misalignment, consequence of the spatial and temporal fluctuations of the source can immediately and significantly aberrate the imaging. Regarding the temporal fluctuations, since the ASE process develops from noise, there may be some concerns on the pointing stability and divergence of a plasma-based source. For comparison, in a fourth-generation accelerator source such as the "LCLS" free-electron laser (XFEL), a pointing stability with a $0.25 \mu \mathrm{m}$ (Table 1) beam precision at the sample plane has been reported [11], which corresponds to a $\mu \mathrm{rad}$ angular tolerance over a $\mathrm{km}$ length system. A footprint reduction in factor 1,000 , from a $\mathrm{km}$-size facility to a tabletop one, brings an advantage in angular tolerance, for a comparable field of view. Indeed, for a $\mathrm{mm}$ shot to shot pointing stability, a mrad tolerance is sufficient for a tabletop system versus a $\mu \mathrm{rad}$ as in the case of the XFEL. Concerning spatial fluctuations, the lack of illumination uniformity of XRL spot may become a reason of concern for applications [12], especially when compared to the excellent figures-of-merit of the synchrotron. Therefore, besides a quantification of the impact of spot uniformity on the imaging quality, optical strategies were developed in this work, in order to compensate for intrinsic plasma-related randomness, and fully exploit the "tabletop potential."

In the case of a spontaneous emission EUV plasma source $(4 \pi s r$ emission), a required component in the microscope is the condenser. The condenser gathers light on a smaller surface and thus enhances the fluence (light per unit surface) to the benefit of illumination contrast. If the fluence is too low, the contrast (or "visibility") is indeed modest, whereas a too strong illumination can generate flare effects, i.e., raising the amplitude baseline of the acquired images, or even damage the sample. The question of the optimal fluence on the sample is still not quantitatively investigated in this context and is subject of 
this work, in order to pinpoint the ideal NA for the condenser. The NA of the condenser and objective are ideally matched, in order to maximize the light collection efficiency into the imaging front end. Thus, the definition of the optimum fluence is functional to define the condenser $\mathrm{NA}$, and then the objective NA.

Nevertheless, the enhancement in brightness that a collimated source brings, e.g., EUV laser [6], as compared to a spontaneous EUV source emitting over $4 \pi s r$ [13], does impact the imaging throughput with as fast as one full image per single shot [14]. Indeed, several groups have shown that a partial enhancement of the coherence has a dramatic effect on the improvement of the visibility $[1,2]$. The issue is here to investigate whether a collimated source has a primary fluence that makes the condenser redundant.

The operation of high peak-brightness imaging sources is in pulsed mode, and to keep the average brightness also high, the pulse repetition rate is another critical parameter to consider. It also influences the number of accumulated counts per image, the statistics and the measurement throughput. The former can be overcome if high peak brightness is there, such that even a single shot is sufficient for a high contrast acquisition as shown previously [15]. If the imaging is done using photo-emission of charged particles (e.g., PEEMS), it is, however, believed that a low peak brightness with high repetition rate is important to mitigate space charge artifacts. Simulations have, however, indicated that for angle-resolved studies, high pump brightness can help to preserve the pristine characteristics due to the generation of a sheath boundary layer in the charged particle bunch [16]. On the other hand, single-shot imaging is advantageous to overcome problems of sample vibrations, as possibly due to instrumentation in the laboratory, e.g., pumps. XRL based on solid-state pump laser technology presently offers repetition rates up to a few Hertz [17, 18]. Research to scale up the repetition rate of XRL toward the $100 \mathrm{~Hz}$, especially in combination with sub-10-nm emission, is ongoing worldwide [17, 19].

Finally, concerning the objective, the use of either multilayer optics or zone plates for short-wavelength imaging suffers from poor efficiency, alignment sensitivity and high cost. Furthermore, laboratory-scale microscopy is difficult to push below the 100-nm resolution, for which reason, lensless methods based on coherent diffraction imaging (CDI) have been proposed by Miao et al. [20-22] and more recently holography techniques have been also used for the same purpose [23]. In order to enable lensless imaging in the laboratory, a tabletop high brightness source with coherence width larger than the sample size is required. Whether the plasma-laser can combine brightness and coherence length is investigated here, and quantitative information of the possible sample sizes for CDI experiments is provided.
As mentioned, the investigations on the source should be combined with those on the imaging optics, in order to match the respective characteristics. Objectives for shortwavelength spontaneous sources have been realized using the (inverse) Schwarzschild design (properly speaking a Cassegrain design), i.e, two multilayer-coated mirrors working as a primary large concave mirror with a central hole and a secondary smaller convex mirror [24]. The classical Schwarzschild alignment of a concave and convex pair addresses third-order aberration issues that a single spherical mirror of large NA suffers from, as shown experimentally before [15]. Few groups using Schwarzschild objectives have achieved high resolution below $100 \mathrm{~nm}[25,26]$. Pros and cons in conjunction with plasma sources are investigated in this work.

The illumination into the primary is annular, since the secondary mirror obscurates the axial region at the end of which is the hole on the primary. Such central obscuration is about $15-20 \%$ depending on the objective's NA. A further side effect of the central obscuration is observed, if illuminated with coherent light, since the border of the secondary mirror will cause diffraction fringes. Therefore, coherent sources would have better imaging performance with objectives without central obscuration. Such alternative designs are considered here as known as "Partial Schwarzschild" [27] or "Yolo" or "Schiefspiegler" objective [28]. The main disadvantage of these designs is that due to the tilting of the mirrors third-order aberrations occur, as shown quantitatively here.

The aim of this work was to provide constraints on the illumination and imaging characteristics of a plasma-based short-wavelength laser using TGRIP and to obtain critical tolerances for a tabletop nano-scale microscope using design-adapted Schwarzschild objectives. The parameters discussed above were analyzed and quantified combining computational studies and experimental data. Given that the observed raw characteristics of a plasma-laser were insufficient to provide reproducible illumination on a microscope entrance pupil, we present technical solutions on pre-pulse delivery and light collimation for fully compensating the EUV laser output randomness and provide stable and homogeneous light filling on the sample.

\section{Materials and methods}

\subsection{EUV laser source}

The experimental measurements were realized at the inhouse Bern Advanced Glass Laser for Experiments ("BeAGLE") EUV laser source. The 1,054 nm Nd:glass laser, with two pre-pulses $(0.5$ and $8 \%$ are the respective amplitudes to the total pump energy) with identical pulse 
duration as the collinear main pulse ( $\tau=1.2 \mathrm{ps}$ ), delivered 2.5-3 J on a tin ( $\mathrm{Sn}$ ) planar target over a $12-\mathrm{mm}$ line focus (GRIP). The results were compared with single orthogonalincidence pre-pulse with a grazing incident main pulse [29] ("classical" transient collisional excitation, TCE). The main advantage of our TGRIP scheme is the precision in adjusting the pre-pulse with the main pulse overlap, even with a tight line focus. The double pre-pulse generates a pre-plasma, that is, the stepwise excited prior to main pulse ignition.

\subsection{Beam characterization}

Figure 1 shows the implemented setup for the measurement of the pointing stability and the divergence in the far field. Nd:Glass laser incises on the Sn-target with an angle of $50^{\circ}$ from the surface. EUV radiation is created and amplified in the plasma column. The radiation propagates to a spherical multilayer mirror, named due to its function "micro-collimator," placed at a distance to the plasma equal to the mirror's focal length. The micro-collimator had a $250 \mathrm{~mm}$ radius of curvature $(R)$. The mirror was coated with a Mo/Y multilayer. The incoming EUV radiation is driven to the CCD by using a turning mirror which was flat and coated with Mo/Y multilayer. The CCD $(9 \mu \mathrm{m}$ pixel size) had a front phosphor screen for EUV-to-visible conversion. In front of the EUV CCD detector, a 150-nmthick layer of zirconium was used to block the off-band radiation. Such setup produced an infinity correction and collimated the incoming XRL pulses, whatever its fluctuating divergence or line-of-sight. The raw image, before the micro-collimator, was captured by a spectrometer. The horizontal axis was the dispersion dimension of the spectrometer. Pointing stability and divergence were measured with and without the micro-collector.

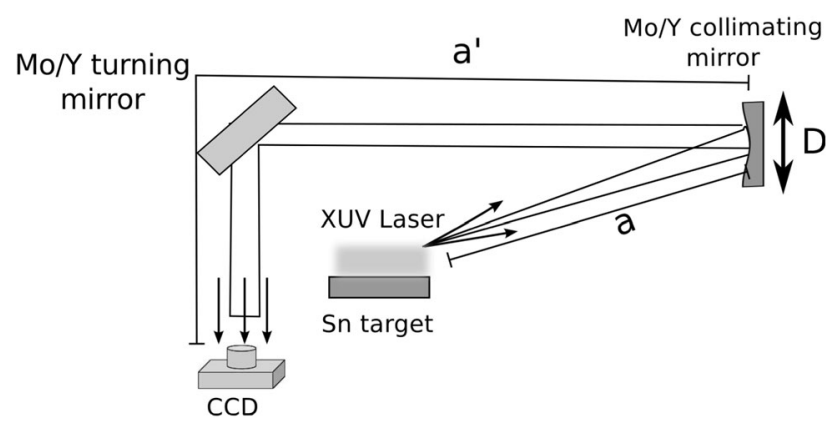

Fig. 1 Experimental setup for far-field characterization of the plasma-driven laser illumination. The optical collimation is obtained by adjusting the position of the collimating mirror to use it as a microcollimator, in order to improve the pointing stability and divergence. The optimum collimation is acquired for distance set as $a=$ $R / 2=250 \mathrm{~mm} / 2$. Sketch not to scale

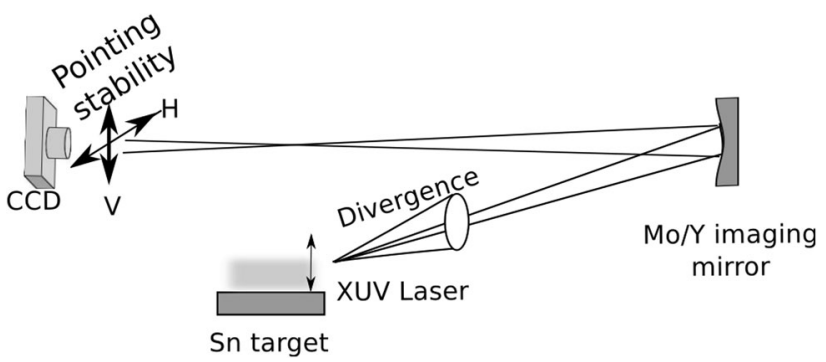

Fig. 2 Experimental setup for near-field characterization of the plasma-driven laser illumination. Sketch not to scale

Figure 2 shows the setup used for near-field imaging. The Nd:Glass laser incises on the Sn-target with an angle of $50^{\circ}$ as above. EUV laser illuminates a spherical multilayer mirror at a distance longer than its focal length thus producing near-field imaging. The image has a magnification of 10 -fold. The same setup was used for the observation of near field with TCE as reported before [29].

\subsection{Spatial coherence measurement}

For the measurement of the coherence of BeAGLE (see above in Sect. 2.1), we based on the Young double-slit experiment. Different double-slit masks were used. Table 2 shows the slit width and the slit separation used. The two pre-pulse schemes (TGRIP and TCE) were compared. Results obtained with the TCE scheme were published before [30]. The visibility $(\gamma)$ to obtain the coherence degree was calculated using the following definition:

$\gamma=\left|\frac{I_{\max }-I_{\min }}{I_{\max }+I_{\min }}\right|$

where $I_{\max }$ and $I_{\min }$ are referred to the maximum and minimum intensity in the diffraction patterns obtained after the illumination on the double-slit masks. Some patterns obtained with the TGRIP are in Fig. 3. The coherence width, $w_{\text {coh }}$, was obtained as: $\frac{\lambda}{\theta}$, where $\lambda$ is $12 \mathrm{~nm}$, the wavelength, and $\theta$ is the angle subtended by the separation

Table 2 Parameters used in the double-slit experiment for the spatial coherence measurement

\begin{tabular}{llllll}
\hline $\begin{array}{l}\text { Number } \\
\text { of slit }\end{array}$ & $\begin{array}{l}\text { Separation } \\
(\mu \mathrm{m})\end{array}$ & $\begin{array}{l}\text { Minimum } \\
\text { width } \\
(\mu \mathrm{m})\end{array}$ & $\begin{array}{l}\text { Maximum } \\
\text { width } \\
(\mu \mathrm{m})\end{array}$ & $\begin{array}{l}\text { Slit } \\
\text { length } \\
(\mathrm{mm})\end{array}$ & Pre-pulse \\
\hline 1 & 9.9 & 3.2 & 3.6 & 3 & GRIP \\
2 & 17.8 & 3.7 & 4.2 & 3 & TCE \\
3 & 19.5 & 4.1 & 4.8 & 3 & GRIP \\
4 & 25.2 & 3.8 & 4.9 & 3 & TCE \\
5 & 33.0 & 4.2 & 5.0 & 3 & TCE \\
6 & 40.6 & 2.8 & 4.1 & 3 & TCE \\
7 & 51 & 3.8 & 4.2 & 3 & TCE/GRIP \\
\hline
\end{tabular}


Fig. 3 Relative coherence width as a function of slit separation for TCE (filled square) pre-pulse scheme. No significant difference was found diamond) and TGRIP (empty between the schemes

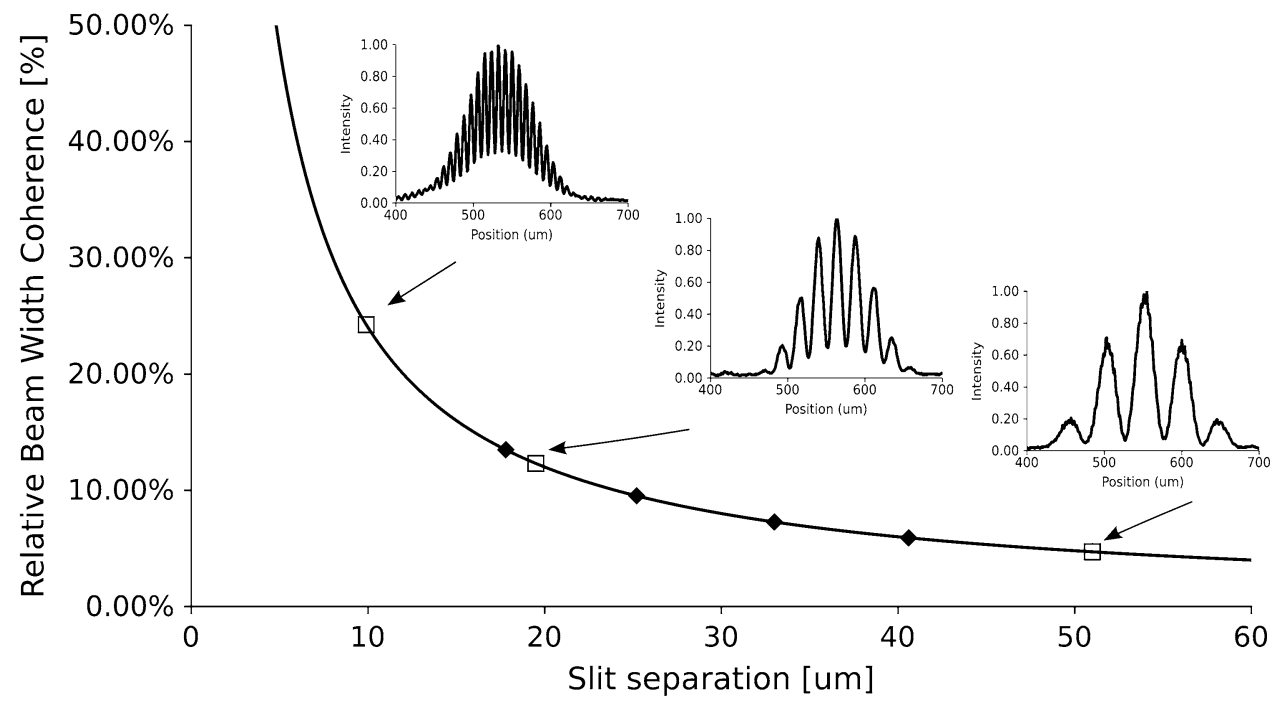

of the slits and the distance to the source. That step is needed to normalize the distance for the measured coherence degree obtained with different setups. The coherence width is considered for the slit separation where $\gamma$ falls to $1 / e$.

\subsection{Fluence}

The signal-to-noise ratio was measured as a function of the illumination fluence. The obtained experimental calibration is shown in Fig. 4, between the illumination (top-axis) and the signal-to-noise ratio (SNR). The observed SNR was fitted as a function of fluence, $F$ (in counts per unit surface) with the following curve:

$S N R=\frac{4096}{1+7 \cdot \exp \left(-4.6 \times 10^{-7} \cdot F\left[\mathrm{cts} / \mathrm{cm}^{2}\right]\right)}$

The amplitude of 4,096 is explained by the 12-bit digitization $\left(2^{12}=4,096\right.$ counts $)$.

\subsection{Modeling of the objective}

The modeling of the Schwarzschild objective was done with OSLO [31], which uses the ray tracing for the analysis of the aberrations. The ray is defined by its entrance pupil coordinates $(x, y)$ and the image coordinates $\left(x_{0}, y_{0}\right)$. The distortions are measured by using the Seidel aberration function [32]. The aberrations are defined according to the ray displacement on the wavefront shape emerging from the exit pupil, and they are expressed in term of an aberration polynomial:

$$
\begin{aligned}
W\left(x, y, x_{0}\right)= & A_{1}+A_{2}+A_{3}+B_{1}+B_{2}+B_{3}+B_{4}+B_{5} \\
& +B_{6}+\ldots
\end{aligned}
$$

where

$$
\begin{aligned}
& A_{1}=a_{1}\left(x^{2}+y^{2}\right) \quad \text { (defocus) } \\
& A_{2}=a_{2} x x_{0} \quad \text { (tilting) } \\
& A_{3}=a_{3} x_{0} \quad \text { (phase shift) } \\
& \left.\begin{array}{l}
B_{1}=b_{1}\left(x^{2}+y^{2}\right) \\
B_{2}=b_{2} x x_{0}\left(x^{2}+y^{2}\right)
\end{array}\right) \\
& B_{3}=b_{3} x^{2} x_{0} \\
& B_{4}=b_{4} x_{0}^{2}\left(x^{2}+y^{2}\right) \\
& B_{5}=b_{5} x x_{0}^{3} \\
& B_{6}=b_{6} x_{0}^{4}
\end{aligned}
$$

$a_{1}$ represents the coefficient of defocus corresponding to the paraxial optics, $a_{2}$ is the coefficient containing the information about the tilting, and $a_{3}$ is the coefficient which refers to a phase shift. The coefficients $b_{1}-b_{6}$ express the wavefront aberrations: spherical, coma, astigmatism, Petzval radius and distortion. The aberrations are consequence of the deviation of the wavefront whose is consequently deteriorated in the quality of the Airy disk. For a discussion where we mention the spherical, coma or astigmatism coefficient, the pupil entrance is expressed in polar coordinates as in [14]. The image of a point which is not limited by diffraction is limited by the aberrations, specified as the ratio of the geometrical spot size and the magnification. The spot size is defined as the root mean square (RMS) spot radius in each main plane (tangential and sagittal). The tolerance for the resolution limited by the aberrations is given by the so-called Strehl ratio, defined as the ratio of the observed peak intensity at the image plane compared to the theoretical maximum peak intensity of a perfect optical system [33]. According to the Maréchal criterion [34], we used the limit of the tolerance at $80 \%$ of the Strehl ratio. For the optimization of the objective, the software uses a standard optimization algorithm called damped least squares [31]. The divergence, pointing 
Fig. 4 Signal-noise ratio as a function of fluence, and illumination (top-axis). Points (filled circle) are experimental data. The fitting is a sigmoid curve $\left(R^{2}=0.94\right)$ given in Eq. 2. Point $(A)$ is the threshold of the linear range, $B$ is the threshold for imaging with SNR $>0.5$ and $C$ is the saturation level. See discussion in the text

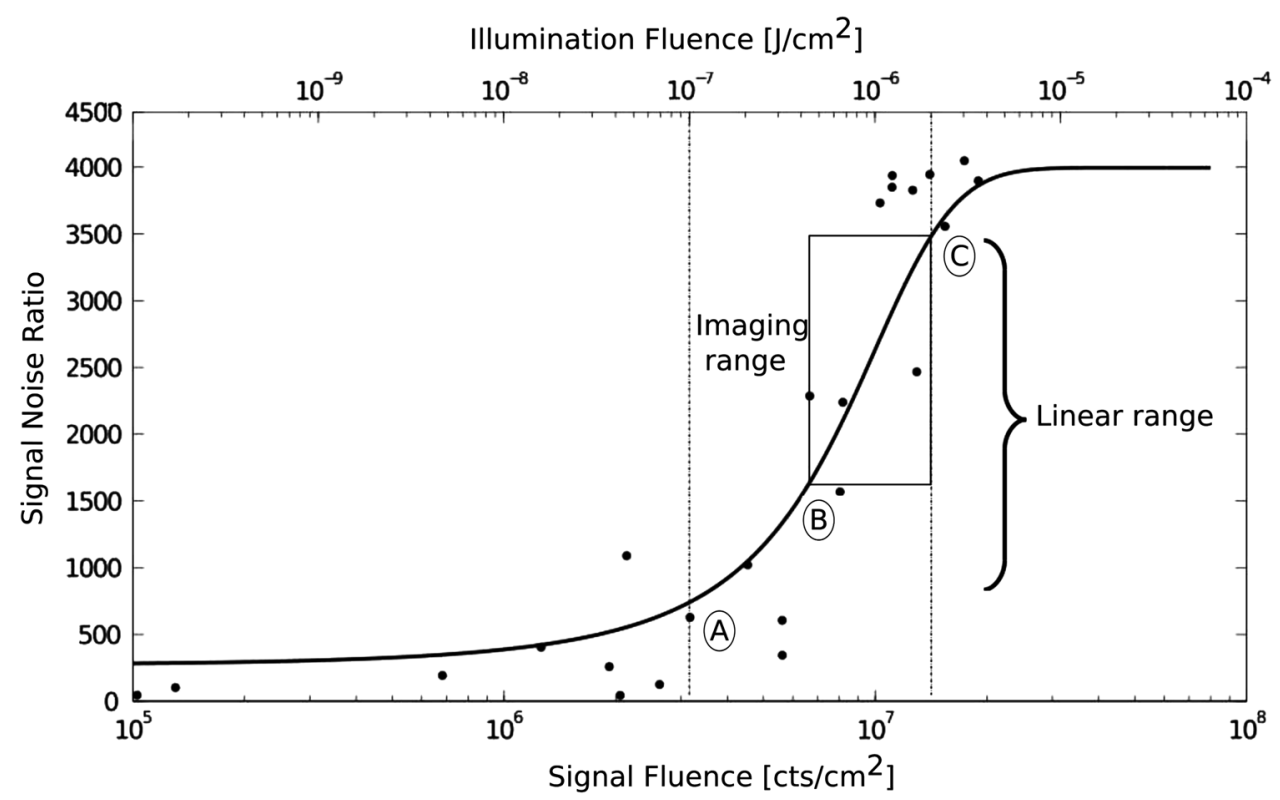

stability and signal-noise ratio were modeled with a selfwritten MATLAB code, which evaluated directly and individually the experimental images obtained in our laboratory. This code gives position coordinates for each pulse. A second self-written code calculates the pointing stability in mrad as the standard deviation of all the coordinates. The divergence was calculated for the horizontal and the vertical axis. Each axis is defined by a so-called quasi-Gaussian profile [35]. The dimensions of the pulses were determined for a width of those profiles at $1 / e^{2}$. The data are post-processed in terms of statistical analysis including the average, the measurement of uncertainty and the standard deviation. The pointing stability was given as the $1 \sigma$ precision. In order to understand the effect of the source profile homogeneity (illumination noise density), we added white Gaussian noise. The inhomogeneity has a Gaussian distribution. We defined the probability of finding a deviation $\delta$ from the mean $(\delta=0)$ as:

$p(\delta)=\frac{1}{\sigma \sqrt{2 \pi}} e^{\frac{\delta^{2}}{2 \sigma^{2}}}$

where $\sigma$ is the standard deviation. The intensity was normalized attributing the maximum intensity (white) to the value 0 and minimum intensity (black) to 255 in the gray scale. Visibilities at different profiles have been calculated using Eq. 1. Four different geometries based on Schwarzschild design were studied and listed here below.

\subsubsection{Classical Schwarzschild}

The classical Schwarzschild is a design with the primary and the secondary mirror having identical center of curvature. By using a primary concave and a secondary convex mirror, the spherical, coma and astigmatism aberrations up to the third order are compensated (See Fig. 5a). For full NA illumination of the primary mirror, a spontaneous source is required, or the expansion of a collimated beam.

\subsubsection{Eccentric Schwarzschild}

The eccentric design is a modification of the above system consisting of offset centers of curvature for primary and secondary mirrors (Fig. 5b). It implies that the object-tocenter-of-curvature distance $(\Delta Q)$ is optimized for superior imaging performance, i.e., minimization of aberration spot size, and for the positioning tolerance.

\subsubsection{Partial Schwarzschild}

Figure $5 \mathrm{c}$ shows the partial Schwarzschild. It uses two spherical mirrors but the primary is offset from the obscuration range and tilted in order to project the incoming pencil of light to the secondary. Both mirrors have common center and the secondary is in the same axis as the object and the image, which results in an illumination cone tilted by the angle $\alpha$ with respect to the optical axis.

\subsubsection{Off-axis Schwarzschild}

The off-axis-illumination Schwarzschild is a special case of the "partial" design. From the point of view of a plasma-laser, the illumination has such small aperture that this design matches with this specification. The "off-axis" 
(a)

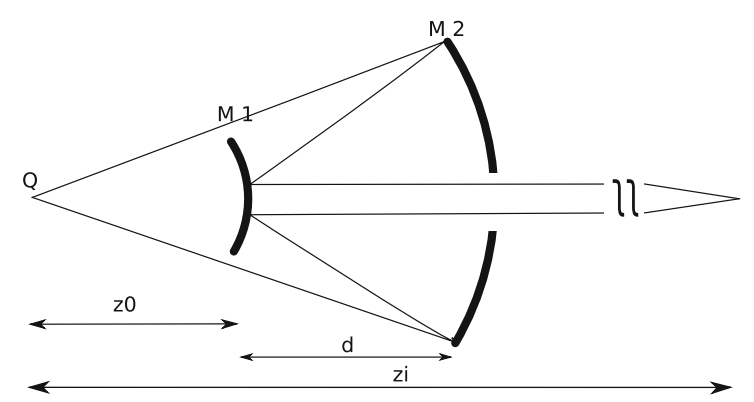

(b)

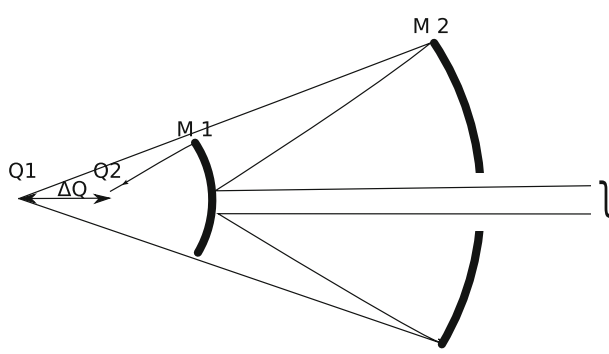

(c)

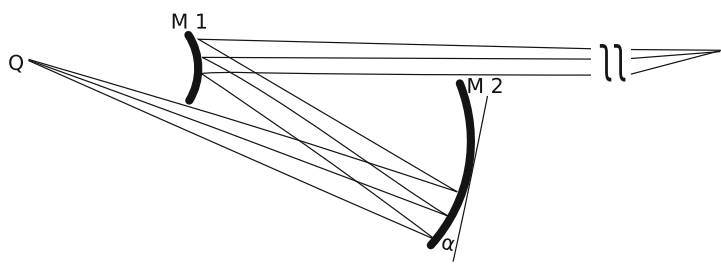

(d)

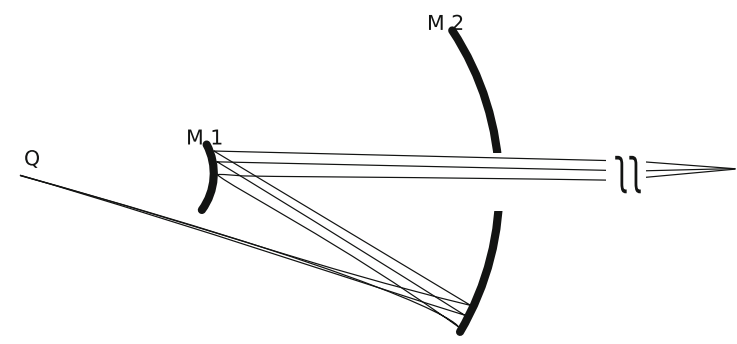

Fig. 5 Sketch of the modified Schwarzschild objectives studied. a Classical Schwarzschild objective. b Eccentric Schwarzschild. c Partial Schwarzschild. d Off-axis Schwarzschild

uses the same configuration as the "classical," but employees only a portion of the primary mirrors (Fig. 5d). This is indeed not a new optical designs but the case of the "classical" illuminated with a laser source.

\section{Results and discussion}

\subsection{Characterization of the EUV laser source}

\subsubsection{TGRIP versus classical TCE pumping}

The pointing stability of a laser is affected by several factors: plasma hydrodynamics, ASE development from noise, energy fluctuations, etc. The unaided plasma-driven laser emission showed an experimental divergence of $5.0 \pm 1.0 \mathrm{mrad}$ (defined as twice the beam NA). Such value would imply a beam diameter of $4-6 \mathrm{~mm}$ over $1-\mathrm{m}$ free propagation up to a microscope entrance pupil. The latter must be filled homogeneously and reproducibly (no shot missing it). The pointing stability of the plasma-laser spot centroid was measured as $0.5 \mathrm{mrad}$ (corresponding to a diameter of $0.5 \mathrm{~mm}$ over $1 \mathrm{~m}$ for the centroid $X / Y$ spread), which is excessive for a stable feeding of the entrance pupil of a microscope. These experimental results were confirmed by a companion computational study published elsewhere [36].

Near-field plasma imaging was carried out to visualize the source spot under the two different pre-pulse schemes explained above, namely TGRIP and classical TCE. In the classical TCE scheme, multiple concomitant hot spots inside the plasma gain-medium contributed to the superposition of contending beamlets ("modes"), with an irregular "multi-mode" spot profile (Fig. 6b). Figure 6a shows that the TGRIP scheme instead converts the spot profile to a more uniform low-mode one (quasi-Gaussian). The improved spot uniformity (low spot noise) is advantageous for imaging applications, which is intuitive, but also confirmed quantitatively in the imaging quality analysis reported below with a Siemens star.

Figure 7 compares the divergence and pointing stability of the two pre-pulse schemes. The divergence was determined as vertical and horizontal principal axes. One notes that our TGRIP scheme gives a factor of 3 more collimated divergence, in both horizontal and vertical directions than the classical TCE scheme. The pointing stability was also improved, by a factor of approx. 2.5, in the TGRIP scheme.

Finally, the results obtained for the spatial coherence measurement of BeAGLE were shown above in Fig. 3. The spatial coherence for TGRIP and TCE once normalized to the same propagation distance ( $1 \mathrm{~m}$ away from the source), show no mismatch of the respective regression curves. This indicates that the pre-pulse scheme has no relevant effect on the coherence degree of the plasma-laser beamlets. However, in the TGRIP, the latter combine in a consistent bundle. Therefore, CDI experiments with sample as wide as tens of a micrometer are possible in a tabletop plasmabased system, which is, however, out of the scope of this paper, and becomes subject of ongoing work.

\subsubsection{Micro-collimator for beam conditioning}

Even considering the spot uniformity improvement discussed with the TGRIP pre-pulse scheme, the obtained line-of-sight (pointing) reproducibility was clearly insufficient for a routine nano-inspection EUV microscope. Therefore, a further technical solution was investigated. In order to improve the pointing stability and collimation of 
(a)

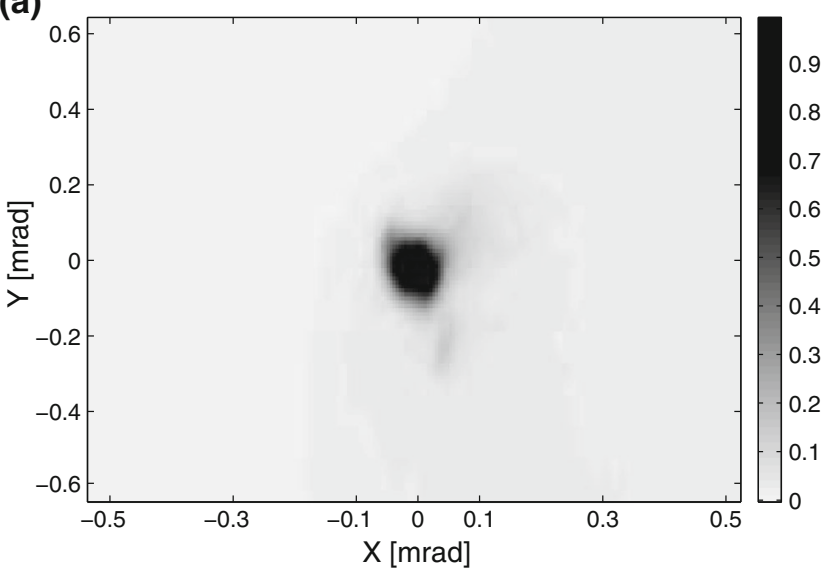

(b)

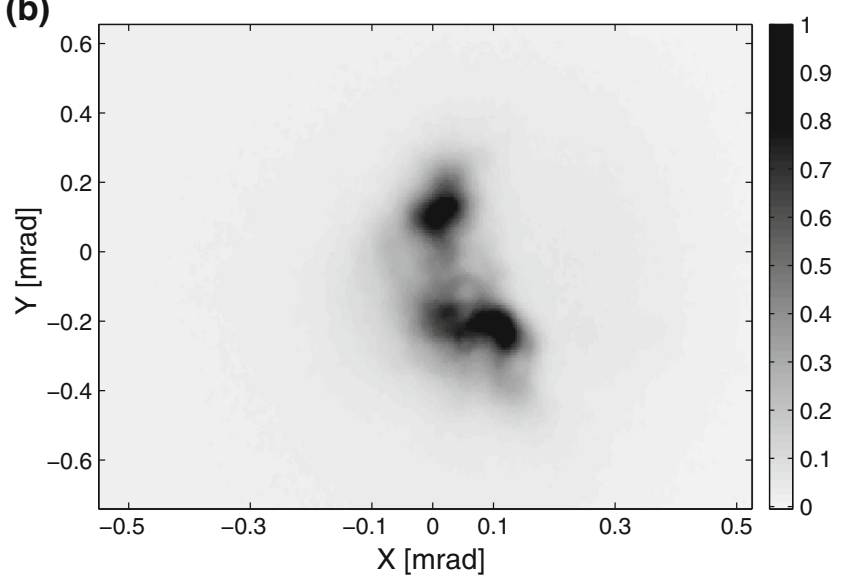

Fig. 6 Near-field illumination spot of the plasma-laser source obtained with a TGRIP b TCE scheme. The comparison shows a more uniform low-mode (quasi-Gaussian) spot for TGRIP than for TCE. See discussion in the text for details

Fig. 7 Pointing stability and divergence for a TGRIP, $\mathbf{b}$ TCE pumping scheme pre-pulse. The TGRIP scheme gives a factor of 3 more collimated divergence than the TCE scheme. The pointing stability was approximately factor of 2.5 improved in the TGRIP scheme (a)

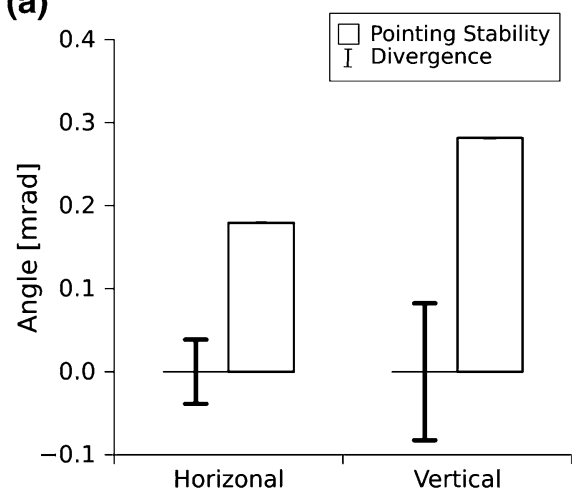

(b)

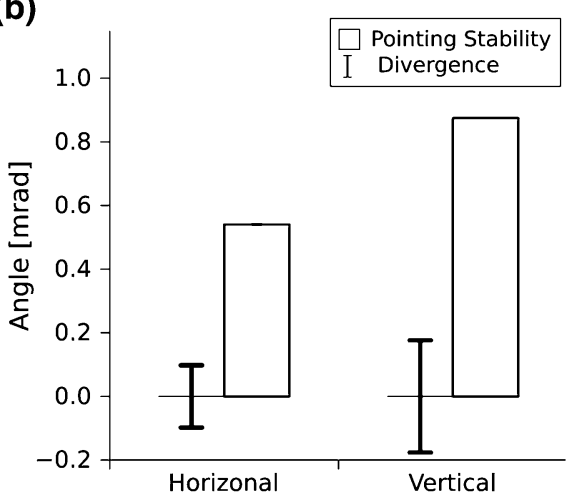

the illumination, the setup was modified implementing an original projection scheme using a micro-collimator with focal length $(f)$ equal to the source distance $(a)$. Figure $8 \mathrm{a}$ shows the calculated divergence of the beam as a function of mirror position as well as the experimental case in our laboratory. One notes that the divergence could be manually optimized down to $0.5 \mathrm{mrad}$ whereas nano-positioning devices would be required for better adjustment. However, given the low curve slope approaching the condition $a=f$, the benefit would be insignificant. The collimated beam was also stabilized in pointing angle because whatever the angular input, the beam was systematically parallelized to the mirror's axis. Indeed, Fig. 8b shows that the inclined illumination of the collimating mirror (on-axis illumination is not possible because it would project the light back on its source) had a limiting value at approx. $4.5^{\circ}$ (in Fig. $8 \mathrm{~b}$ data are in mrad), with the same considerations made above about alignment tolerances.

The collimated beam was directed on a CCD for the farfield mapping of the spot. Figure 9 shows that the pointing stability improved by a factor of 5 (from approx. 0.5 to 0.1 $\mathrm{mrad}$ ) and the divergence a factor of 10 (from $5 \pm 1.0$ to
$0.5 \pm 0.1 \mathrm{mrad}$ ) when our collimation scheme was implemented. These results showed an increase in source stability and also brightness, by means of reducing the étendue, since brightness is defined as power/étendue. Étendue is the area of the source per solid angle at the entrance pupil of the objective. The new correction can be theoretically understood by considering the Gaussian mirror equation [37], with the following given specifications:

$\frac{1}{f=125 \mathrm{~mm}}=\frac{1}{a=125 \mathrm{~mm}}-\frac{1}{a^{\prime} \rightarrow \infty}$

where $a$ is the distance from the object to the mirror, $a^{\prime}$ is the distance from the object to the detector, and $f$ is the focal length of the mirror equal to $R / 2$, with $R$ the radius of curvature of the micro-collimator. The formula can be recast to explain the functionality of the micro-collimator as follows:

$\beta=\left(\frac{1}{f=150 \mathrm{~mm}}-\frac{1}{a=125 \mathrm{~mm}}\right) \cdot D$

where for small divergence angles, $-a^{\prime} \approx D / \beta$ can be used, with $\beta$ the divergence (twice the NA) and $D$ the diameter of 
Fig. 8 Calculated a divergence and $\mathbf{b}$ pointing angle of the plasma-laser beam as a function of the micro-collimator mirror position. The experimental value obtained at our facility by manual adjustment is indicated by the star
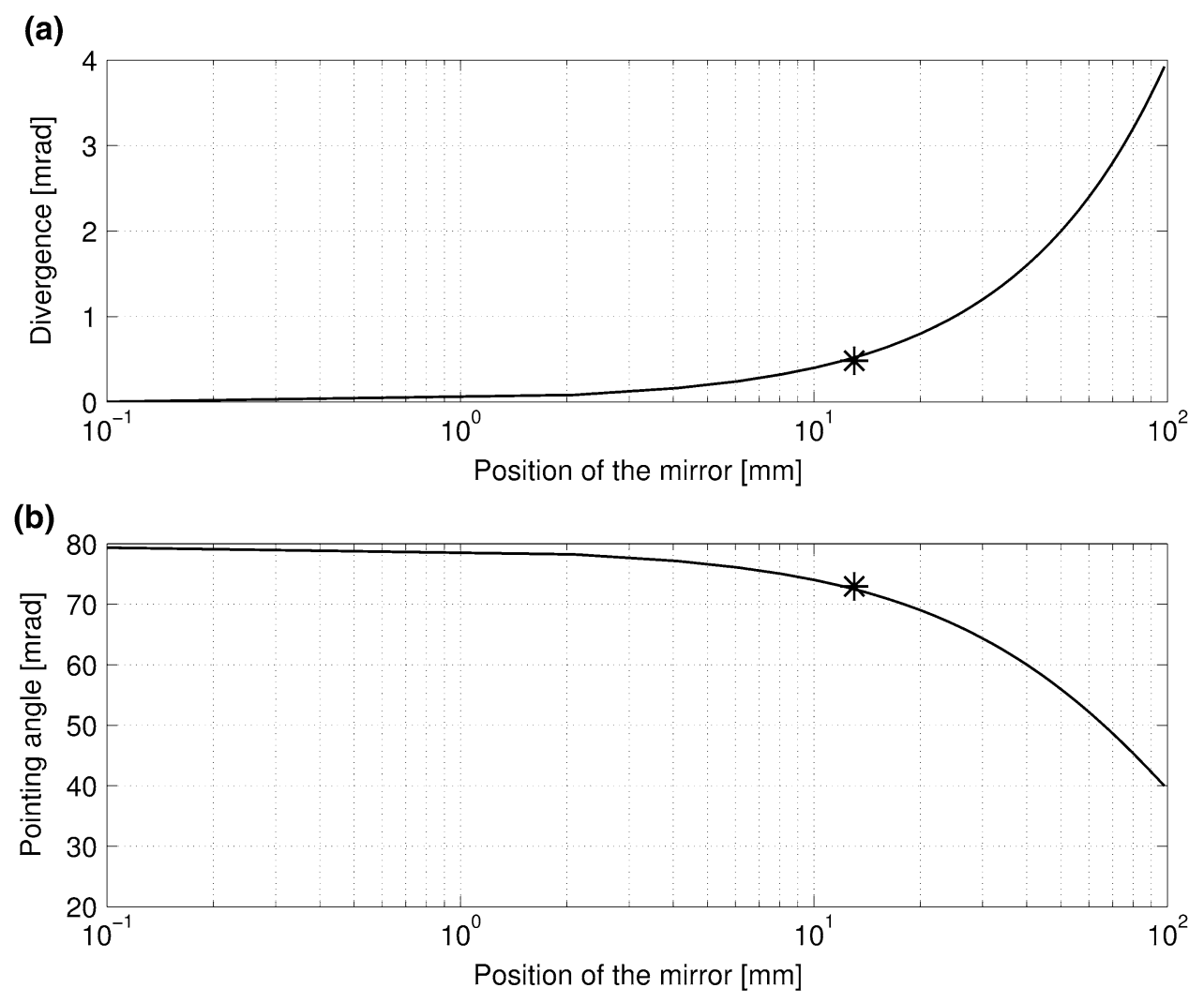

Fig. 9 a Plasma-laser beam divergence and $\mathbf{b}$ pointing stability with and without the micro-collimator. The figure a shows an improvement of a factor 10 in the divergence and b a factor of 5 in the pointing stability (a)

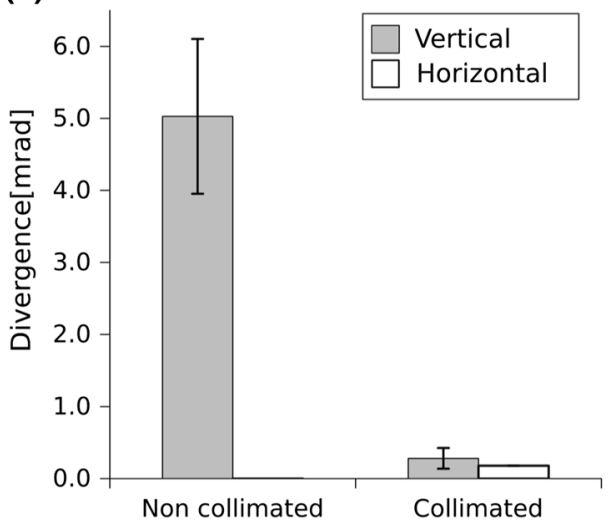

(b)

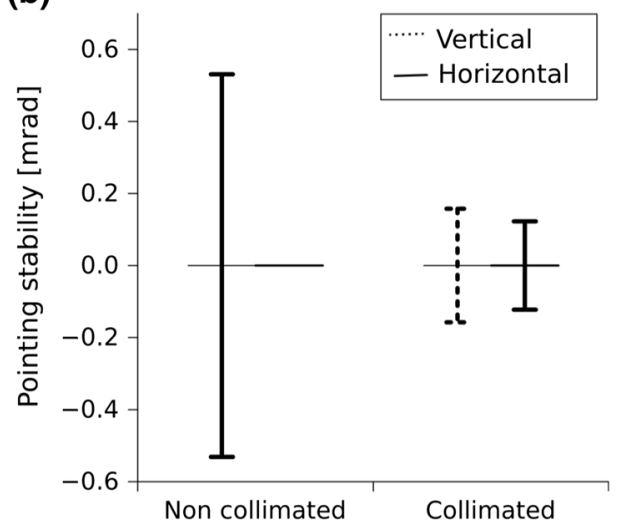

the illuminated part on the micro-collimator. In Fig. 8a, one observes that the divergence decreases when the distance between the plasma source and the micro-collimator $(a)$ is approaching to the focal length of the collimator.

\subsubsection{Optimum fluence for quality nano-imaging}

The obtained control on the peak brightness of the EUV laser questioned whether a condenser was at all required as in [15], or as it is customary for spontaneous EUV plasma sources. To address this point, we analyzed the results plotted in Fig. 4, showing the output signal-to-noise ratio versus the input fluence. The dark current in our experimental data was less than 100 counts. The fitting carried out using Eq. 2 showed an agreement with the experimental data points as high as $R^{2}=0.94$. Since the output of the plasma-laser is in the range of a few $\mu \mathrm{J}$ and the area of the CCD chip is of the order of $1 \mathrm{~cm}^{2}$, the raw (no optics in-between) illumination fluence is within the linear range, i.e., $\simeq 10^{-6} \mathrm{~J} / \mathrm{cm}^{2}$. In Fig. 4 the linear range, marked with two dashed lines between points $\mathrm{A}$ and $\mathrm{C}$, starts at $10^{-7}$ $\mathrm{J} / \mathrm{cm}^{2}$ up to $3^{-6} \mathrm{~J} / \mathrm{cm}^{2}$. Accounting for a $10-20 \%$ imaging optics throughput, the illumination, however, may degrade below the linear range (approx. $10^{-7} \mathrm{~J} / \mathrm{cm}^{2}$ ). To overcome 
such threshold, if it is not possible by controlling the beam size to maximum 1-2 $\mathrm{mm}$ at the sample position, one can accumulate signal with multiple-shot imaging.

Figure 10 provides a computational analysis of the imaging performance of a quarter of a Siemens star reference sample, as a function of sample-related illumination amplitude (net signal) and illumination uniformity (spot noise). The illumination amplitude is investigated at reference values of $10 \%$ (bottom row), $25 \%$ (central row) and $100 \%$ (top row), for the case of null background (Fig. 10a). The illumination noise is investigated at reference values of $0 \%$ (RHS column), $10 \%$ (center column) and $90 \%$ (LHS column). The visibility $(\gamma)$ of the Siemens star spokes is computed following Eq. 1 and is reported at the corner of each snapshot. The total illumination amplitude on the detector (net sample-related signal + background) is given at the bottom of each box. Figure 10b provides the same analysis for the case with flare, i.e., with background equal to $50 \%$ of the dynamic range. It is to remind that $100 \%$ corresponds to full dynamic range.

The scientific discussion focuses on the optimization of the illumination amplitude (fluence) and reduction in flare, but as shown here is critical to understand the effect of the illumination homogeneity. Indeed, this provides a rationale for the importance for imaging applications of the improved spot homogeneity following our TGRIP scheme, as shown above. The baseline level affects the off-field image, the peak-to-valley contrast, as well as the residual dynamic range. However, for a virtually flat-field illumination, a non-zero baseline level can enhance the visibility of nano-scale features. The latter can remain unresolved only if spot noise degrades the illumination. Therefore, a threshold for high quality nano-imaging is set at point $\mathrm{B}$ in Fig. 4, namely when $S N R>0.5$ of linear range.

From the presented computational analysis, one concludes that the imaging optimization benefits more from enhanced illumination homogeneity than comparable increase in spot intensity. Indeed, one can compare the corresponding quarter Siemens star visibility degradation with spot noise and note how an inhomogeneous (noisy) spot degrades the imaging, even at high illumination amplitude. The experimental images of the Siemens star with a single concave-mirror objective [14] showed a visibility of $\gamma=0.68$ at the amplitude of $90 \%$ of full dynamic range. Such results can be improved by increasing the signal uniformity, rather than suppressing the background leaking between the Siemens star spokes.

\subsection{Schwarzschild-derived objective designs for nano-scale imaging}

The spatial resolution is well known to be limited by diffraction, which motivates the use of multilayer optics that can be operated with short-wavelength sources. It is,

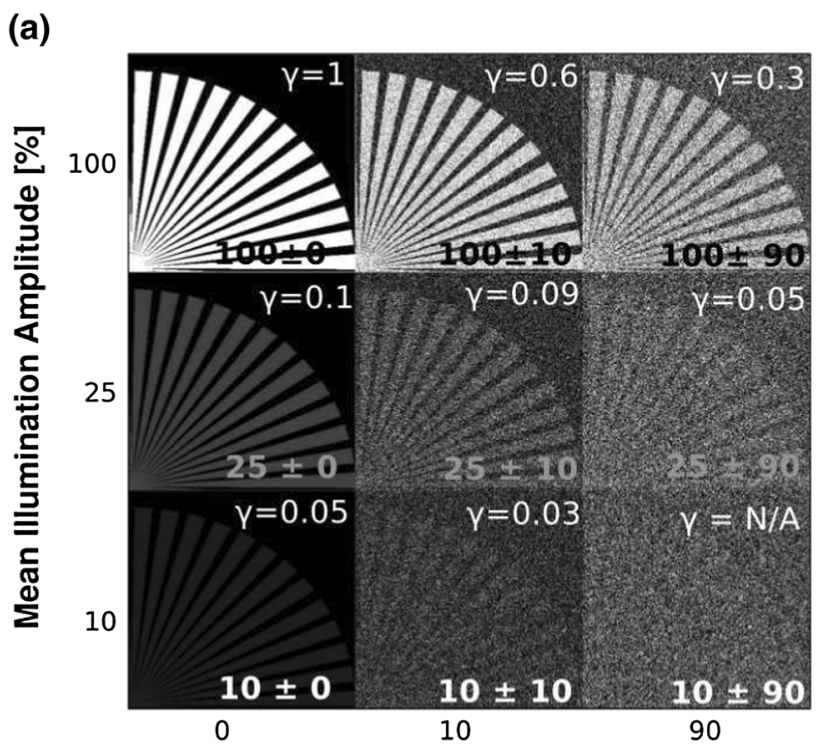

Illumination Noise [\%]

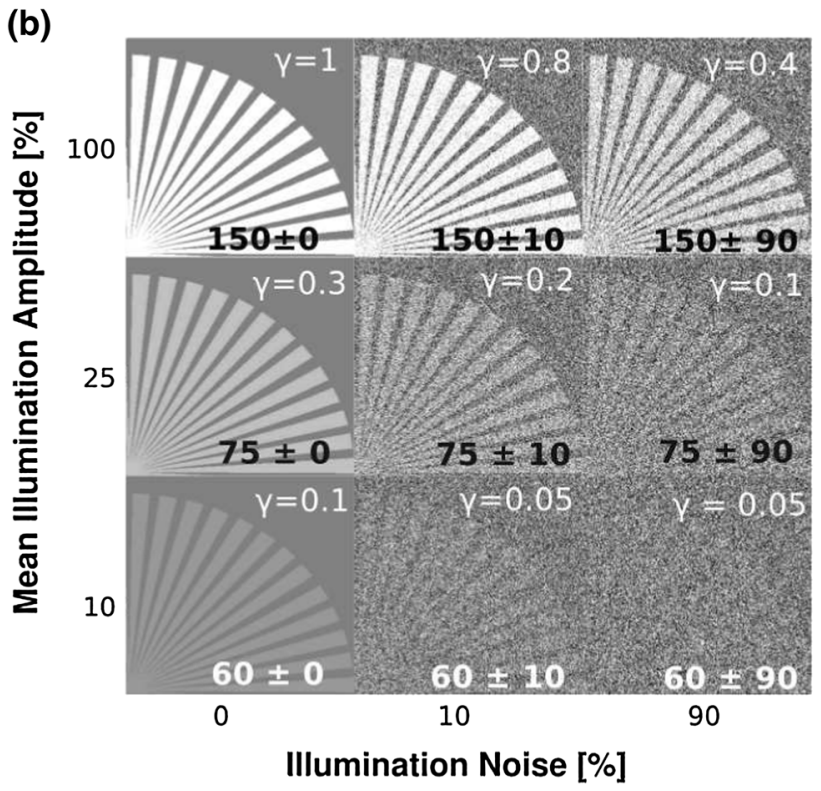

Fig. 10 Calculated imaging performance on a quarter Siemens star by changing the illumination noise at three steps of $0,10,90 \%$ ( $x$-axis) and the mean of the net illumination amplitude on the spokes at 10,25 and $100 \%$ ( $y$-axis). The spokes visibility $(\gamma)$ obtained for each box is given as well as the total illumination amplitude arriving at the detector. All values are in percent with respect to the full dynamic range (saturation level is at $100 \%$ )

however, to be reminded the importance to choose a high NA to enlarge the high frequency cutoff of collected wave vectors. However, large NA can be prone to more significant third-order aberrations, as discussed above. Figure 11 compares the performance of the Schwarzschild designs introduced in the Sect. 2.5. The Strehl ratio of $80 \%$ is at the cutoff of the resolution limited by diffraction and the resolution limited by aberrations. The latter changes in the 
(a)

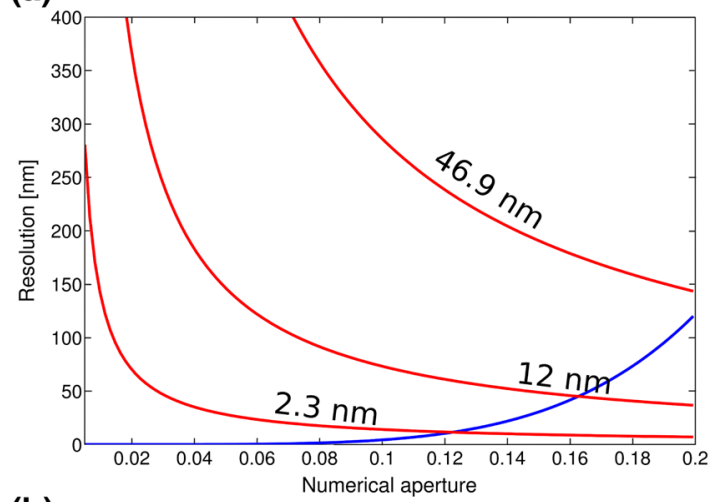

(b)

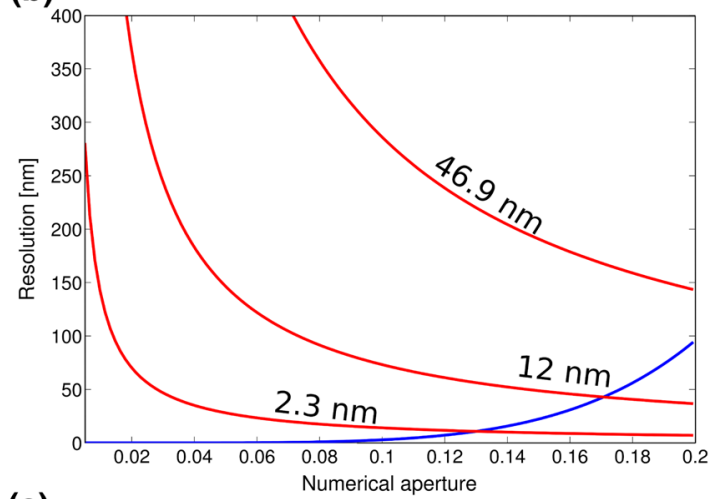

(c)

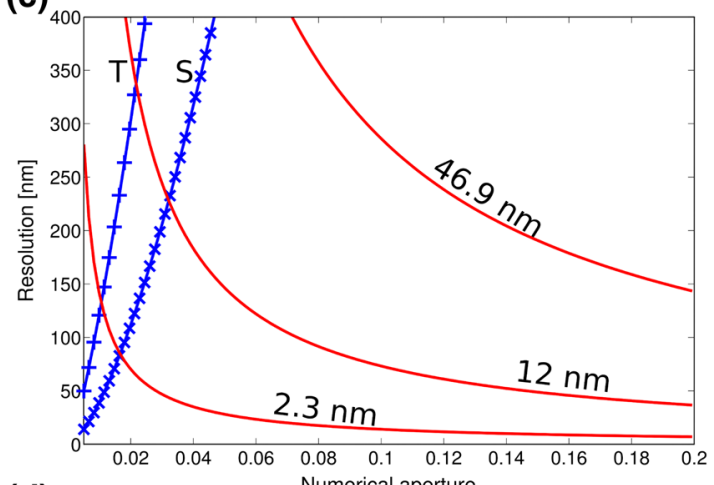

(d)

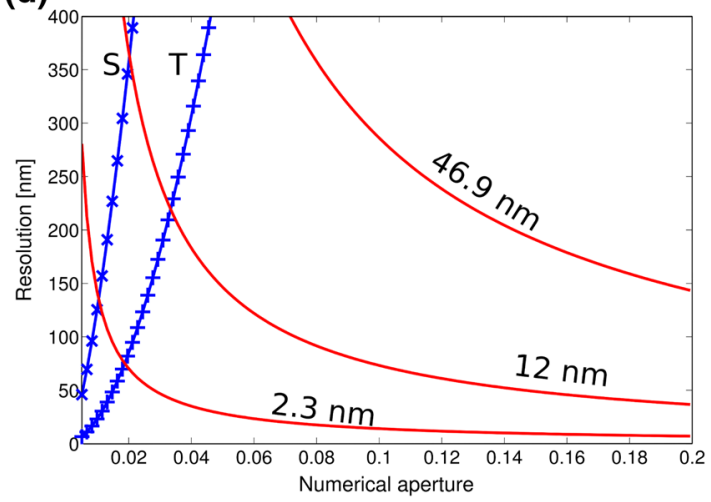

four configurations of the Schwarzschild with the position and inclination of primary or secondary mirror. Magnifications above $15 \times$ in Schwarzschild are possible for a ratio
Fig. 11 Calculated resolution as limited by diffraction (red lines) at three wavelengths, i.e., $12 \mathrm{~nm}$ (BeAGLE), $2.3 \mathrm{~nm}$ (water-window radiation) and $46.9 \mathrm{~nm}$ (capillary-discharge laser), and as limited by third-order aberrations (blue lines) as a function of the numerical aperture for the four studied objectives: a classical, b eccentric, $\mathbf{c}$ partial and $\mathbf{d}$ off-axis Schwarzschild. In $\mathbf{a}$ and $\mathbf{b}$ astigmatism is fully corrected for such as no difference is found in the sagittal and tangential planes. In $\mathbf{c}$ and $\mathbf{d}$, the symbol $T$ refers to the tangential and the $S$ refers to the sagittal plane

of primary to secondary mirror radii of curvature $\left(R_{1} / R_{2}\right)$ between 2.5 and 3 [38] (Fig. 12). Based on this criterion, we choose the radii of curvature for all the designs as $R_{1}=100$ and $R_{2}=36 \mathrm{~mm}(r=2.8)$ for primary and secondary mirror, respectively, which correspond to a magnification of $30 \times$.

The classical Schwarzschild has an aperture limited by the diffraction and aberration resolution of 0.16 (Fig. 5a) which corresponded to 45-nm resolution. The produced obscuration by the secondary mirror is $15 \%$. The reflectance of each mirror is $45 \%$; thus, the total reflectance of the Schwarzschild, taking in account the obscuration too, is $17.2 \%$. Increasing the NA involves increasing the diameter of primary mirror or decreasing the distance $z_{0}$ between the object and the mirror (see Fig. 5). Both alternatives imply also a potential increase in the aberrations, specially for classical and eccentric designs. Figure 11 shows that the resolution and numerical aperture are linked with an inverse relationship. For NA higher than 0.15 , there is no significant increase in the resolution. Resolution limited by aberration links also with the aperture.

The eccentric Schwarzschild was adjusted to separation of the centers of curvature of the primary and secondary mirror in the range of $150-200 \mu \mathrm{m}$, which allows to increase the NA to 0.17 by keeping the same Seidel coefficients but not the obscuration which increases to $18 \%$. The free third-order aberration of both optics, classical and eccentric, can be understood in Fig. 13a, b where is shown the wavefront and the ray analysis. The wavefront indicates a peak-to-valley of 0.125 and $0.110 \mathrm{~mm}$, respectively, at the entrance pupil. In the ray analysis, we observe that the aberrations are not significant for $0.5-\mathrm{mm}$ field of view. In order to have a good compromise of obscuration and resolution, we propose a 0.15 eccentric model, which has not a significant improvement in resolution $(50 \mathrm{~nm})$ and it is only limited by diffraction.

The partial Schwarzschild has higher spherical aberrations for the same NA as the classical or eccentric models. The Airy disk is not symmetric with respect to the optical axis, and the aberrations have a notably difference in the horizontal and the vertical direction. For instance in a $N A=0.025$, the tangential resolution is $325 \mathrm{~nm}$ whereas 


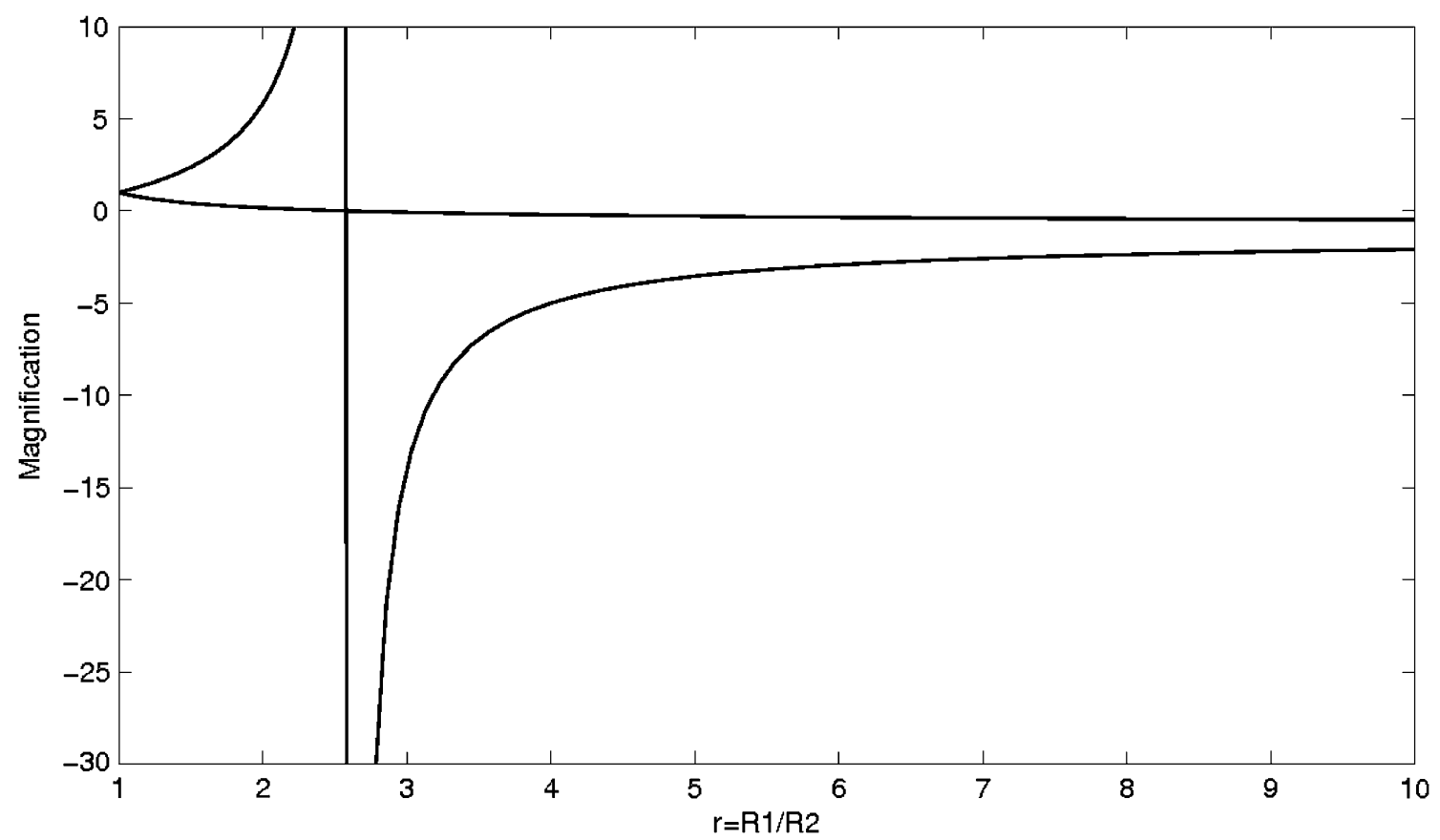

Fig. 12 Aplanatic condition for Schwarzschild as a function of magnification and mirror's radii ratio $r=R 1 / R 2$. The simulation agrees with results from [38]

the sagittal resolution is $230 \mathrm{~nm}$. In Fig. 13c, we observe this asymmetry, which is basically astigmatism, in the wavefront surface. The value of the coefficients for the third order coma and astigmatism is smaller than $\left|10^{-6}\right|$. The major aberration contribution is done by orders higher than fifth and by the spherical aberration.

The off-axis Schwarzschild's simulations showed a resolution of $250 \mathrm{~nm}$ for a non-significant spherical aberrations. The illumination incises on the primary mirror out of the optical axis. The objective can be employed with a laser source by using a scanning beam while the "classical," "eccentric" and "partial" designs are more relevant with spontaneous sources since they can be used with a full-field beam. Coma aberration is observed in this design (see Fig. 13d). The value of the third order coefficients is approximately $10^{-5}$ for coma and astigmatism. They both are generated as a result of the inclined incident beam on the optics. The aperture of 0.03 is perfectly compatible with BeAGLE since the results of the measurement had shown smaller divergence.

\section{Conclusions}

The TGRIP plasma gain-medium generation scheme was shown to give a factor of 3 more collimated laser output than a classical TCE short-wavelength plasma-laser as well as better uniformity. The pointing stability was approximately factor of 2.5 also improved in the TGRIP scheme. Additionally, we have demonstrated a simple method to control both divergence and pointing stability using a micro-collimator. The insertion of a micro-collimator in the setup improved by a factor of 5 (from approximately 0.5 to $0.1 \mathrm{mrad}$ ) the pointing stability and the divergence a factor of 10 (from $5 \pm 1.0$ to $0.5 \pm 0.1 \mathrm{mrad}$ ). The coherence degree found from the visibility modulation was shown to remain unchanged in the TGRIP versus TCE schemes. The measured coherence length of several tens of micrometers allows for coherent diffraction imaging, in order to have a lensless sub-50-nm nano-imaging tool. Ongoing work in this field will be subject of a future publication. The measured fluence to noise relationship showed that optimum brightness in the linear range is within the specifications of plasma-laser output. Within this range, the contrast may be degraded by the illumination inhomogeneity (spot noise). Experimental images obtained by a single EUV laser shot showed to be at optimum theoretical conditions.

The development of the appropriate optics for EUV plasma-laser involved the study of the obscuration and the aberrations. Classical and eccentric Schwarzschild are free of third-order aberration. High resolution smaller than $60 \mathrm{~nm}$ is possible with both optics. However, the position of the secondary mirror produces an obscuration of 15 and 
(a)

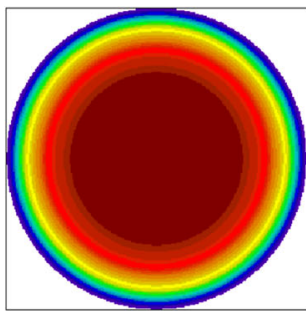

Peak: $0.000 \mathrm{~mm}$

(b)

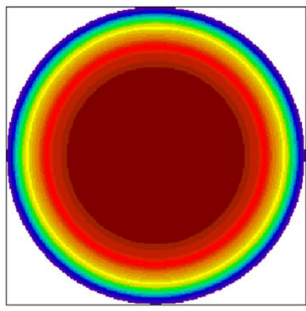

Peak: $0.000 \mathrm{~mm}$

(c)

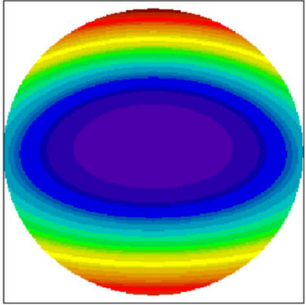

Peak: $3.750 \mathrm{~mm}$

(d)

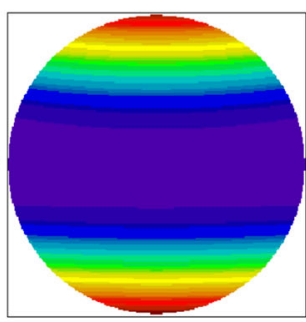

Peak: $12.650 \mathrm{~mm}$

Valley: $-0.240 \mathrm{~mm}$
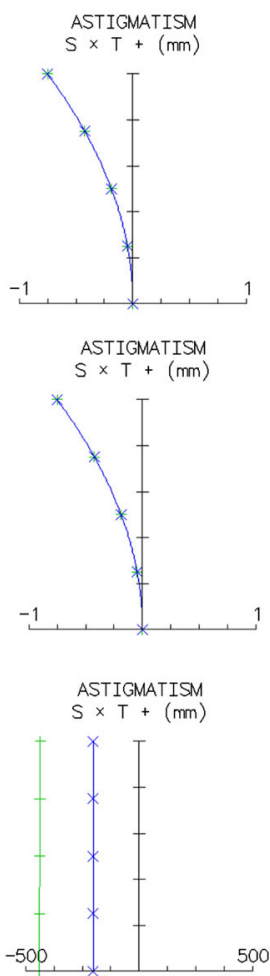

ASTIGMATISM

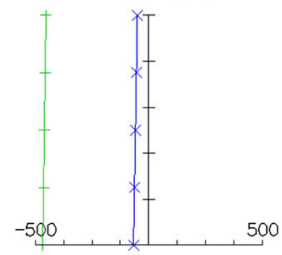

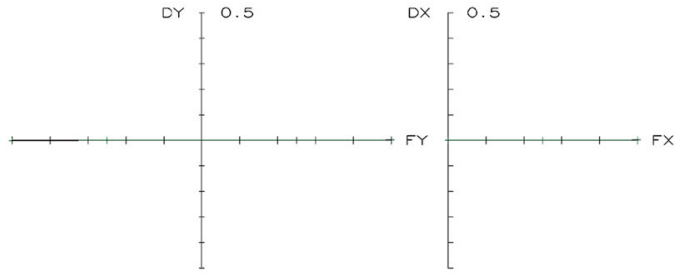
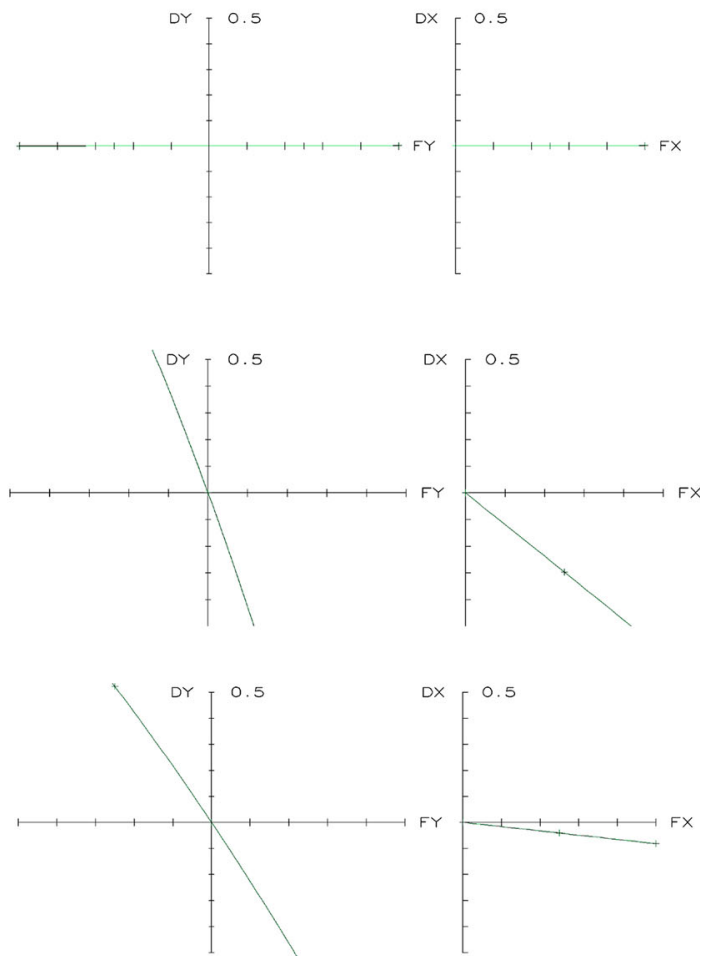

Fig. 13 Wavefront (left), astigmatism analysis (center) and ray analysis (right) for the a classical, b eccentric, c partial and $\mathbf{d}$ offaxis Schwarzschild. $\mathbf{a}$ and $\mathbf{b}$ are free of third-order aberrations. We

Table 3 Comparison of the critical parameters for the different microscope designs, which are studied here

\begin{tabular}{lllll}
\hline & Classical & Eccentric & Partial & Off-axis \\
\hline NA & 0.16 & 0.17 & 0.02 & 0.02 \\
Resolution (nm) & 45 & 43 & 365 & 330 \\
Obscuration (\%) & $15 \%$ & $18 \%$ & None & None \\
Magnification & $30 \times$ & $31 \times$ & $25 \times$ & $30 \times$ \\
Efficiency $(\%)$ & $17.2 \%$ & $16.8 \%$ & $20.25 \%$ & $20.25 \%$ \\
\hline
\end{tabular}

$18 \%$. The partial Schwarzschild is devoid of such problem of obscuration at the cost of adding the spherical aberrations and off-axis introduces coma. By restricting the numerical aperture, the two latter can be used in EUV laser microscopes by using a scanning beam. Table 3 summarizes the main parameters of the Schwarzschild studied in this work. observe in c coma and astigmatism aberration due to the inclination of the rays through the partial and off-axis optics, $\mathbf{d}$ is affected by coma

Acknowledgments The authors are grateful to I. Artyoukov (Lebedev Institute), J. Fessler (University of Michigan), and our colleagues F. Staub, L. Masoudnia, J.E. Balmer, and Th. Feurer (University of Bern) for discussions. The present work was supported by the Swiss National Science Foundation under the Grant Number PP00P2-133564/1.

\section{References}

1. P. Wachulak, A. Bartnik, H. Fiedorowicz, Proceedings of the School on Short-wavelength Imaging Spectroscopy, 2012, ed. by D. Bleiner, SPIE Digital Library, Bern, 10-11 Sept. 2012

2. F. Brizuela, Y. Wang, C.A. Brewer, F. Pedaci, W. Chao, E.H. Anderson, Y. Liu, K.A. Goldberg, P. Naulleau, P. Wachulak, M.C. Marconi, D.T. Attwood, J.J. Rocca, C.S. Menoni, Opt. Lett., 34(3), 271-273 (2009)

3. K.A. Goldberg, I. Mochi, J. Vac. Sci. Technol., B 28(6), C6E1-10 (2010)

4. H. Merbold, A. Bitzer, T. Feurer, Opt. Express 19, 8 (2011)

5. D. Strickland, G. Morou, Opt. Comm. 56, 221-229 (1985) 
6. J. Balmer, D. Bleiner, F. Staub, Micro Nanolith MEMS MOEMS, 11(2), 021119 (2012)

7. D. Ursescu, B. Zielbaver, Th. Kühl, P. Neumayer, G. Pert, Phys. Rev. E 75, 045401 (2007)

8. A. Klisnick, P. Zeitoun, D. Ros, A. Carillon, P. Fourcade, S. Hubert, G. Jamelot, C. Lewis, A. MacPhee, R. O'Rourke, R. Keenan, P. Nickles, K. Janulewicz, M. Kalashnikov, J. Warwick, J. Chanteloup, A. Migus, E. Salmon, C. Sauteret, J.P. Zou, J. Opt. Soc. Am. B 17(6), 1093 (2000)

9. J. Dunn, R. Keenan, V.N. Shlyaptsev, SPIE International Society for Optical Engineering Conference on Soft X-ray Lasers and Applications VI 5919, 2005

10. M. Grünig, C. Imesch, F. Staub, J.E. Balmer, Opt. Commun. 282, 267-271 (2009)

11. J.L. Turner, R. Akre, A. Brachmann, F.-J. Decker, Y.T. Ding, P. Emma, Y. Feng, A.S. Fisher, J.C. Frisch, A. Gilevich, P. Hering, K. Horovitz, Z. Huang, R.H. Iverson, D. Kharakh, A. Krasnykh, J. Krzywinski, H. Loos, M. Messerschmidt, S.P. Moeller, H.-D. Nuhn, D.F. Ratner, T.J. Smith, J.J. Welch, J. Wu, Proceedings of Particle Accelerator Conference, THP168, 2011

12. B. Ehlers, Y. Platonov, B. Verman, J. Rodriguez, personal comment

13. W.T. Silfvast, IEEE J. Quant. El. 358, 700 (1999)

14. D. Bleiner, F. Staub, J. Balmer, Proceeding of SPIE, vol 8140 , 2011

15. D. Bleiner, F. Staub, V. Guzenko, Y. Ekinci, J. Balmer, Opt. Commun. 284(19), 4577-4583 (2011)

16. D. Bleiner, J. Balmer, Appl. Phys. Lett. 98, 181501 (2011)

17. B.A. Reagan, K.A. Wernsing, A.H. Curtis, F.J. Furch, B.M. Luther, D. Patel, C.S. Menoni, J.J. Rocca, Opt. Lett. 37(17), 3624-3626 (2012)

18. J. Tümmler, R. Jung, H. Still, P. Nickles, W. Sanwer, Opt. Lett. 34, 1378 (2009)

19. G. Korn, A.Thiss, H. Stiel, U. Vogt, M. Richardson, Th. Elsässer, M. Faubel, Opt. Lett. 27, 866 (2009)

20. J. Miao, P. Charalambous, J. Kirz, D. Sayre, Nature 400, 342-344 (1999)

21. K.S. Raines, S. Salha, R.L. Sandberg, H. Jiang, J.A. Rodriguez, B.P. Fahimian, H.C. Kapteyn, J. Du, J. Miao, Nature, 463, 214-217 (2010)

22. A. Ravasio1, D. Gauthier, F.R.N.C. Maia, M. Billon, J-P. Caumes, D. Garzella, M. Geleoc, O. Gobert, J-F. Hergott, A-M. Pena, H. Perez, B. Carre, E. Bourhis, J. Gierak, A. Madouri, D. Mailly, B. Schiedt, M. Fajardo, J. Gautier, P. Zeitoun, P.H. Bucksbaum, J. Hajdu, H. Merdji, Phys. Rev. Lett.103, 028104 (2009)

23. F. Capotondi, E. Pedersoli, M. Kiskinova, A.V. Martin, M. Barthelmess, H.N. Chapman, Opt. Express 20, 22 (2012)
24. Y. Horikawa, S. Mochimaru, Y. Iketaki, K. Nagai, SPIE 1720, 217 (1992)

25. K. Hamamoto, Y. Tanaka1,T. Yoshizumi1, Y. Fukushima, H. Shiotani, N. Sakaya, M. Hosoya, T. Shoki, T. Watanabe, H. Kinoshita, Proc. SPIE 6151, 615119 (2006)

26. L. Juschkin, R. Freiberger, Proc. SPIE 7360, 736005 (2009)

27. W.T. Silfvast, L. Sun, J. E. Harvey, University of Central of Florida Orlando, Technical report, AN: ADA318404, 1996

28. L. Jones, Handbook of Optics, Ch.18, vol II, ed. by M. Bass, E.W. Van Stryland, D.R. Williams, W. Wolfe, 2nd edn (1995)

29. F. Staub, M. Braud, J.E. Balmer, J. Nilsen, S. Bajt, Appl. Phys. B 78, 7-8 (2004)

30. M. Braud, Doctoral Dissertation. IAP University of Bern, Switzerland, 2003

31. OSLO Version 5 Optics Reference, Sinclair Optics (1997)

32. H.A. Buchdahl. Optical Aberration Coefficients (Dover Publications, New York, 1968)

33. W.J. Smith, Modern Optical Engineering, SPIE Spress, Ch. 15, 4th edn, 2007

34. W.B. Wetherell, Proceedings of SPIE 0028, Instrumentation in Astronomy I, vol 45 (1972)

35. A. Ritucci, G. Tomasselti, A. Reale, F. Flora, L. Mezi, Phys. Rev. A 70, 023313 (2004)

36. L. Masoudnia, D. Bleiner, Laser Phys. 23, 056003 (2013)

37. E. Hecht, Optics, 3th edn. (Addison Wesley, Reading, MA, 1998)

38. I. Artyoukov, K. Krymski, Opt. Eng. 39(8), 2163-2170 (2000)

39. A.R. Präg, T. Mocek, M. Kozlová, B. Rus, K. Rohlena, Eur. Phys. J. D. 26 59-65 (2003)

40. J.J. Rocca, Y. Wang, M.A. Larotonda, B.M. Luther, M. Berrill, D. Alessi, Opt. Lett. 30, 2581-2583 (2005)

41. Y. Liu, M. Seminario, G. Tomasel, C. Chang, J.J. Rocca, D.T. Attwood, Phys. Rev. A 63, 033802 (2001)

42. J. Filevich, J.J. Rocca, M.C. Marconi, R.F. Smith, J. Dunn, R. Keenan, J.R. Hunter, S.J. Moon, J. Nilsen, A. Ng, V. Shlyaptsev, App. Optics 43, 19 (2004)

43. F. Brizuela, C. Brewer, S. Fernandez, D. Martz, M. Marconi, W. Chao, E.Anderson, A. Vinogradov, I. Artyukov, A. G Ponomareko, V.Kondratenko4, D. Attwood, K. Bertness, N. Sanford, J. Rocca, C. Menoni, J. Phys. 186 (2009)

44. I.A. Vartanyants, A. Singer, A. Mancuso, O. Yefanov, A. Sakdinawat, Y. Liu, E. Bang, G.J. Williams, G. Cadenazzi, B. Abbey, H. Sinn, D. Attwood, K.A. Nugent, E. Weckert, T. Wang, D. Zhu, B. Wu, C. Graves, A. Scherz, J.J. Turner, W.F. Schlotter, M. Messerschmidt, J. Lüning, Y. Acremann, 8 P. Heimann, D.C. Mancini, V. Joshi, J. Krzywinski, R. Soui, M. Fernandez-Perea, S. Hau-Riege, A.G. Peele, Y. Feng, O. Krupin, S. Moeller, W. Wurth, Phys. Rev. Lett. 107, 144801 (2011) 